Article

\title{
Functional Insights into the Roles of Hormones in the Dendrobium officinale-Tulasnella sp. Germinated Seed Symbiotic Association
}

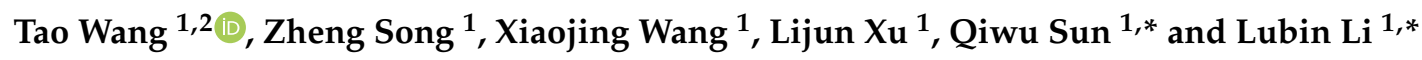 \\ 1 State Key Laboratory of Forest Genetics and Tree Breeding, Key Laboratory of Silviculture of the State \\ Forestry Administration, Research Institute of Forestry, Chinese Academy of Forestry, Beijing 100091, China; \\ wangtao5757@126.com (T.W.); songzheng566@163.com (Z.S.); wangxjwork@163.com (X.W.); \\ xulijun19935@126.com (L.X.) \\ 2 Beijing Botanical Garden, Beijing 100093, China \\ * $\quad$ Correspondence: sqw@caf.ac.cn (Q.S.); lilubin@126.com (L.L.); Tel.: +86-10-6288-9664 (Q.S.); \\ $+86-10-6288-8687$ (L.L.)
}

Received: 8 October 2018; Accepted: 16 October 2018; Published: 6 November 2018

\begin{abstract}
Dendrobium is one of the largest genera in the Orchidaceae, and D. officinale is used in traditional medicine, particularly in China. D. officinale seeds are minute and contain limited energy reserves, and colonization by a compatible fungus is essential for germination under natural conditions. When the orchid mycorrhizal fungi (OMF) initiates symbiotic interactions with germination-driven orchid seeds, phytohormones from the orchid or the fungus play key roles, but the details of the possible biochemical pathways are still poorly understood. In the present study, we established a symbiotic system between D. officinale and Tulasnella sp. for seed germination. RNA-Seq was used to construct libraries of symbiotic-germinated seeds (DoTc), asymbiotic-germinated seeds (Do), and free-living OMF (Tc) to investigate the expression profiles of biosynthesis and metabolism pathway genes for three classes of endogenous hormones: JA (jasmonic acid), ABA (abscisic acid) and SLs (strigolactones), in D. officinale seeds and OMF under symbiotic and asymbiotic conditions. Low concentrations of endogenous JA, ABA, or SLs were detected in the D. officinale-Tulasnella symbiont compared with the asymbiotic tissues. Gene annotation results suggest that the expression of DEGs (differentially expressed genes) related to JA and ABA biosynthesis from $D$. officinale were down-regulated, while most of the key DEGs related to SL biosynthesis from $D$. officinale were up-regulated in the symbiotic germinated seeds compared with the asymbiotic germinated seeds. Moreover, in the OMF, we found a significantly up-regulated differential expression of the JA and ABA biosynthesis-related genes in the symbiotic interaction, with the opposite expression trends to those found in Dendrobium. This indicates that Dendrobium seed symbiotic germination may be stimulated by the apparent involvement of the OMF in the production of hormones, and relatively low concentrations of endogenous JA, ABA, or SLs might be maintained to promote the growth of the D. officinale-Tulasnella symbiotic protocorm-like body. These results will increase our understanding of the possible roles played by endogenous hormones in the regulation of the orchid-fungus symbiosis.
\end{abstract}

Keywords: Dendrobium officinale; Tulasnella sp.; symbiosis; endogenous hormones

\section{Introduction}

In the majority of orchid species, seed germination is largely dependent on mycorrhizal fungi under natural conditions because of the lack of nutritional reserves [1]. Symbiotic seed germination, which is essential for orchid propagation and reintroduction, can be used for the conservation of the Dendrobium species in their natural habitats [2]. The process of orchid seed germination under natural 
conditions is complex and unique, and involves various physiological and biochemical processes such as colonization, plant growth stimulation, and the plant response to the fungus [1]. Although successes in orchid seed germination are regularly reported in the literature, it is important to note that when comparing the asymbiotic and symbiotic germination of the same species, the rates of orchid seed germination, development of the protocorms, and growth is usually better in the latter $[3,4]$. Many studies have shown that orchid mycorrhizal fungi (OMF) promote metabolic processes in orchid seeds $[4,5]$. Although orchid seeds contain very low nutrient reserves, recent analyses of the unique properties of protocorms have shown that during symbiotic seed germination, the expression of putative genes involved in metabolism, transcriptional regulation, and signal transduction pathways is upregulated [5-7]. Miura et al. (2018) examined an orchid-mycorrhizal symbiosis by analyzing the transcriptome of Bletilla striata associated with Tulasnella sp. at an early developmental stage, and the essential genes required for the establishment of a mutualistic relationship with AM fungi and/or rhizobia in most terrestrial plants were identified from B. striata [8]. These studies indicate that the symbiotic association formed from the germinated seed is able to interact with mycorrhizal fungi.

When mycorrhizal fungi undergo symbiotic interactions with orchids, phytohormones produced by the orchid and/or the microbial partners could play key roles [9-11]. The symbiosis between Cymbidum goeringii and a Rhizoctonia-like mycorrhizal fungi causes the release of hormones, which can promote the growth of $C$. goeringii seedlings [9]. The results of comparative transcriptomic and proteomic analyses suggested that an ABA receptor protein, PYR1, showed a significantly higher expression during the early symbiotic germination stages (when the embryo swells, enlarges, and emerges from the seed coat) in D. officinale [10]; the developmental stages are defined according to Stewart et al. [12]. The role of JA signaling is well known in plant-pathogen interactions [11]. A recent study also detected higher levels of lipoxygenase in Oncidium sphacelatum at the green protocorm stage of seed development with Ceratobasidium sp., and this could possibly result in an enhanced JA biosynthesis in the green protocorms with the fungal symbiont [6]. These studies indicate that the functions of the growth-inhibiting hormones ABA and JA could be important for symbiotic seed germination and growth of Dendrobium.

In higher plants, ABA is derived from the oxidative cleavage of carotenoids. Isopentenyl diphosphate (IPP) formed in the 1-deoxy-D-xylulose-5-phosphate (DXP) pathway is converted to geranylgeranyl pyrophosphate (GGPP), from which carotenoids are synthesized $[13,14]$. The carotenoid $\beta$-carotene is hydroxylated to form the xanthophyll zeaxanthin, which is further converted into ABA by a series of enzymes, such as zeaxanthin epoxidase (ZXE) and short-chain dehydrogenase/reductase (SDR). It has been reported that the symbiotic fungi, including OMF, can also produce compounds that are similar to the plant hormome ABA, although studies have shown that the ABA biosynthetic pathways seem to differ between higher plants and fungi [15]. Fungi synthesize ABA via a direct pathway from isopentenyl diphosphate (IDP) and farnesyl diphosphate (FDP), which are derived from the mevalonic acid (MVA) pathway [16,17]. After a series of cyclization, isomerization, and oxidization reactions, ABA is synthesized from farnesyl pyrophosphate (FPP). Labeling experiments have been performed to characterize the ABA biosynthesis pathway in Botrytis cinerea, Cercospora rosicolla, and some other species of fungi [18-20]. Previous studies have shown that $1^{\prime}, 4^{\prime}$-trans-diol-ABA is the main precursor of ABA in B. cinerea and that $\triangle B C A B A 4$ transformants accumulate $1^{\prime}, 4^{\prime}$-trans-diol-ABA [17]. The $B c A B A 4$ gene encodes a putative SDR (short-chain-type dehydrogenase/reductase) which is involved in the ABA biosynthesis in Arabidopsis [16].

The JA biosynthesis pathways of plants and OMF are more similar. JA synthesis is initiated in the plastid by the oxygenation of linoleic or $\alpha$-linolenic acid by lipoxygenases (LOXs) to form (13S)-hydroperoxy linoleic or $\alpha$-linolenic acid. This is then followed by the first committed step of JA biosynthesis catalyzed by allene oxide synthase (AOS) that converts 13-HPOT to a highly reactive allene oxide, which is converted to cis-(+)-12-oxophytodienoic acid by allene oxide cyclase (AOC). cis-(+)-OPDA is then transported to the peroxisome, where 12-oxophytodienoate reductase 3 (OPR3) and three rounds of $\beta$-oxidation convert it to JA [21]. 
Previous studies have shown that plants generally produce multiple strigolactone (SL) species which are released from the roots and induce germination in seeds of the Striga and Phelipanche species in the soil [22,23]. SLs or their biosynthetic precursors act as plant hormones to inhibit shoot branching in plants [24], which was discovered in studies using a series of enhanced shoot branching mutants [22-25]. SLs also function as host recognition signals for AM (arbuscular mycorrhizal) fungi, as well as branching factors in AM fungi [22,23]. A hypothetical SL-biosynthetic pathway was initially proposed, with $\beta$-carotene as a substrate for carotenoid-cleavage dioxygenase enzymes (CCD7 and CCD8, which act sequentially in the pathway). Further investigation of the MAX1 gene, which encodes a cytochrome P450 in the CYP711A1 clade, acting downstream to CCD8 and of its orthologs in Arabidopsis and rice revealed that this gene is expressed in all vascular tissues, and functions only in the late steps of SL synthesis [26]. D14/DAD2 (a probable strigolactone esterase) may be a putative SL receptor involved in SL signaling pathway regulation. Although it has been hypothesized that SL phytohormones have important functions during plant-fungus interactions and symbioses [23], little is known about the role of SLs in the symbiotic associations between orchids and OMF.

These phytohormones are well known to control plant development and to trigger important signaling events [15]. Moreover, plant hormones produced by mycorrhizal fungi have been shown to improve the growth of medicinal orchids [4]. In addition, the possible biosynthesis pathways for plant and fungal hormones in the orchid-fungal symbiosis are poorly understood at present. The manner in which these molecules favor the invasion of plant tissues and the development of fungi inside plant tissues is also largely unknown. To this end, we performed comparative transcriptomic and hormone profiling between symbiotically and asymbiotically germinated $D$. officinale seeds in an effort to gain possible functional insights into the biosynthesis and metabolism of ABA, JA, and SLs in the regulatory module in the Dendrobium-Tulasnella symbiosis. We also predicted some unigenes involved in phytohormone production by the symbiotic fungus Tulasnella sp., and these genes had different expression profiles in symbiotic and asymbiotic germinated seeds.

\section{Results}

\subsection{Detecting Mycorrhizal Fungus Colonization in Germinated Seeds of D. officinale}

Both morphological observation and Trypan blue staining, which showed that the intracellular fungal hyphae stained blue in the symbiotic germinated seeds (DoTc; Figure 1), indicated that a symbiotic relationship had been established between $D$. officinale germinated seeds and Tulasnella sp., while in the group of seeds germinated without fungi with a low germination rate $(0.1 \%)$, we did not find hyphae, and these were therefore defined as asymbiotic germinated seeds (Do).
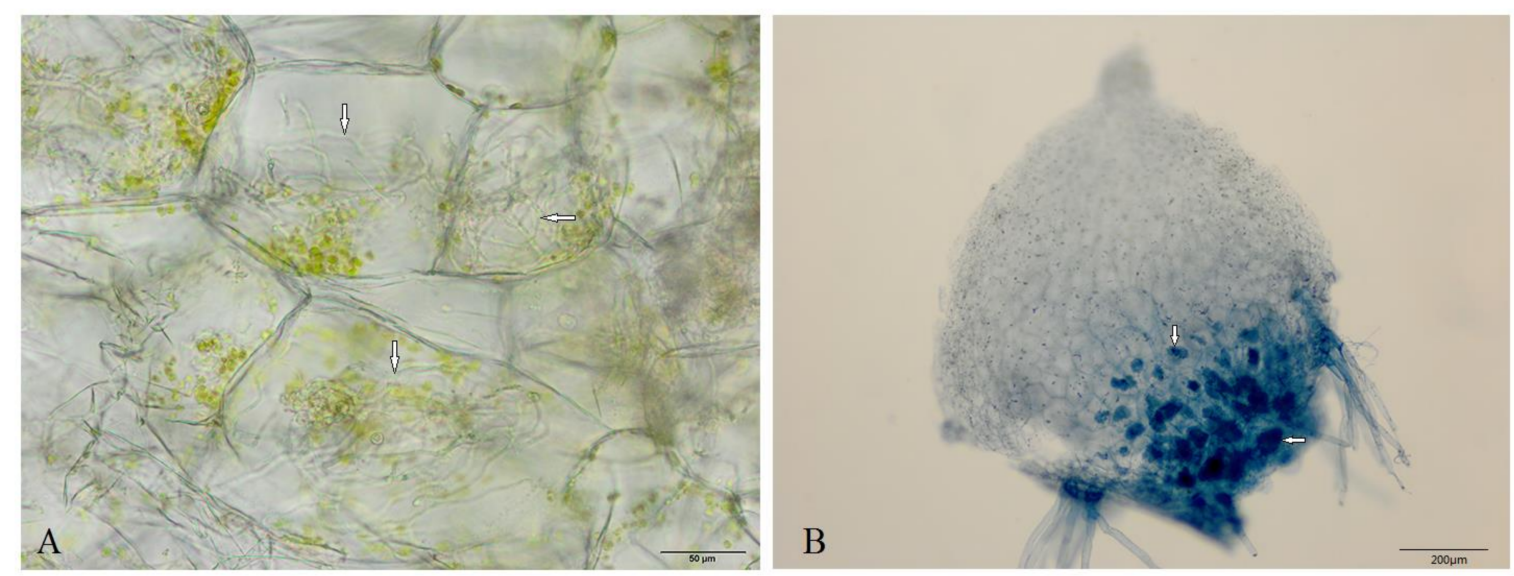

Figure 1. The morphological observation of intracellular hyphae in the symbiotic germinated seeds of $D$. officinale. The microscopic observation of intracellular hyphae in a free-hand section (A) of germinated seeds were stained blue following Trypan blue staining (B). The arrows indicate the symbiotic mycelia. 


\subsection{Transcriptome Sequence Assembly and Annotation.}

To globally characterize the transcriptome of D. officinale and its Tulasnella endosymbiont with enhanced sequence coverage, nine cDNA libraries were constructed using RNA extracted from DoTc, Do, and free-living Tulasnella mycelium (Tc). In total, 667,270,886 Illumina paired-end raw reads were generated. The raw reads are available in the NCBI SRA database under the accession number SRP136098. After discarding the adaptor and low-quality sequences, we obtained 642,119,290 clean reads (accounting for $96 \mathrm{~Gb}$ of DNA sequence). The percentages of clean reads among the raw tags (Q20) ranged from $95.52 \%$ to $96.11 \%$ (Table S1). The cleaned reads of D. officinale from the DoTc and Do libraries were then mapped to the D. officinale genome database [27] with $45.7-50.27 \%$ and $60.62-61.04 \%$ of the reads in the DoTc and Do libraries, respectively, mapping to the genome (Table S2). The novel genes annotated in Dendrobium officinale are shown in Table S3.

The de novo assembly of the clean reads was performed and resulted in 50,597 unigenes that ranged from 201 to 12,137 bp in length, with an N50 length of $1488 \mathrm{bp}$ in the Tulasnella transcriptome. The size distribution of the transcripts and unigenes in Tulasnella is shown in Table S4. The transcriptome assembly produced a substantial number of large unigenes: 15,254 unigenes were >1000-bp in length (Table S4). All assembled unigenes (Table S5) were annotated using BLASTX searches against the NR, NT, KO, SwissProt, PFAM, GO, and KOG databases (Table S6). In total, there were 1637 (3.23\%) unigenes that were annotated in all databases, and 35,616 (79.39\%) that were annotated in at least one database (Table S6), providing significant BLAST results. Among the annotated unigenes, 23,758 (46.95\%) showed significant similarity to known proteins in the $\mathrm{Nr}$ database, 23,532 unigenes $(46.50 \%)$ in the GO database, and 9105 unigenes $(17.99 \%)$ in the KOG database (Table S6).

\subsection{Differentially Expressed Genes in Symbiotic and Asymbiotic Germinated Seeds and Mycorrhizal Fungi}

To compare the differences in gene expression between the symbiotic germinated seeds, asymbiotic germinated seeds, and mycorrhizal fungi, we compared the transcriptome profiles between the symbiotic and asymbiotic germinated seeds (DoTc vs. Do) and symbiotic and asymbiotic mycorrhizal fungi (DoTc vs. Tc). A total of 3367 DEGs (differentially expressed genes) were obtained that included 2230 up-regulated and 1137 down-regulated genes in the DoTc vs. Do comparison (Figure 2A and Table S7), and 7720 differentially expressed unigenes that included 7028 up-regulated and 692 down-regulated unigenes in the DoTc vs. Tc comparison (Figure 2B and Table S8).
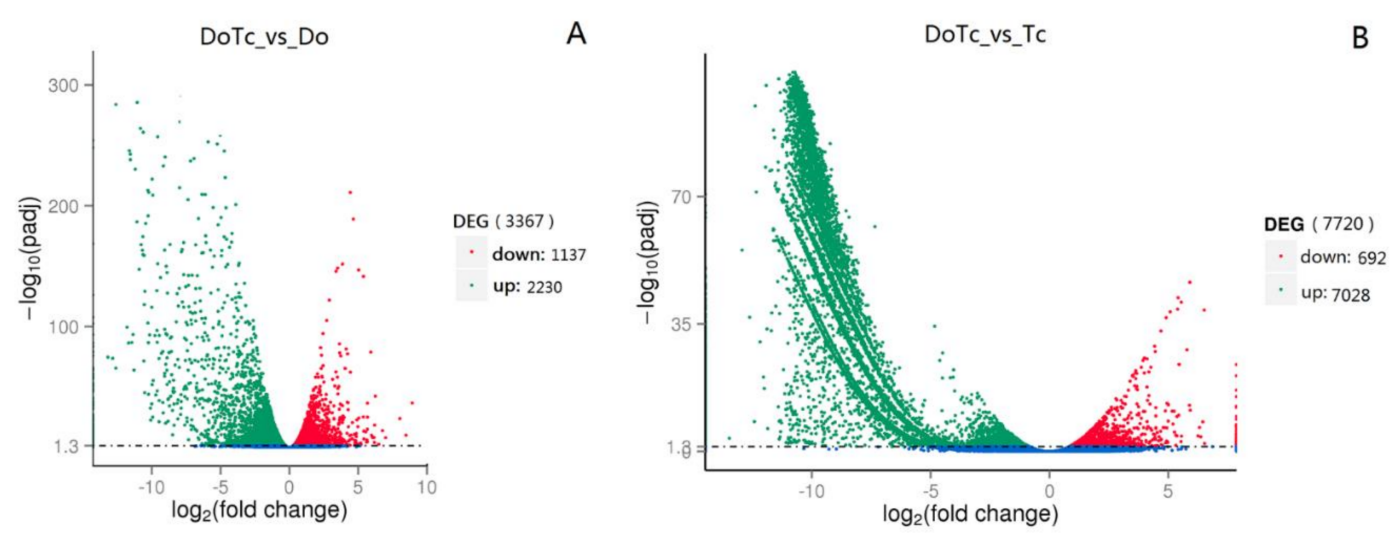

Figure 2. The distribution of differentially expressed genes (DEGs) in symbiotic and asymbiotic germinated seeds. (A) DEGs originating from D. officinale in the symbiotic germinated seeds (DoTc vs. Do), and (B) DEGs originating from OMF (orchid mycorrhizal fungi) in the symbiotic germinated seeds (DoTc vs. Tc). Genes in which the relative level of expression was down-regulated are shown in red, and those that were up-regulated are shown in green. 
The annotation results suggest that orchid seed germination stimulated by the fungal association apparently involves plant hormone signal transduction, plant-pathogen interaction, and metabolic pathways from D. officinale that were obviously up-regulated (Figure S1) in the symbiotic germinated seeds (DoTc vs. Do). However, in the mycorrhizal fungus, the expression of genes for proteins implicated in the ribosome, proteasome biosynthesis, and protein processing in the endoplasmic reticulum were found in the symbiotic interaction (Figure S1, DoTc vs. Tc).

It is well known that plant hormones have important functions during plant-fungal interactions. In the present study, we found that DEGs related to hormone pathways show a significant differential expression (Figure S1A); we thus sought to gain possible functional insights into the biosynthesis and metabolism of three types of plant hormones-JA, ABA, and SL-in the regulatory module of the Dendrobium-Tulasnella symbiosis. In the ABA biosynthesis pathway, the expression of ZXEs and $S D R s$ in D. officinale was significantly down-regulated in the symbiotic germinated seeds compared with the asymbiotic germinated seeds. However, the possible ABA biosynthesis-related short-chain type dehydrogenase/reductase-encoding gene BCABA4 [16] and the P450 monooxygenase-encoding genes $B C A B A 1 / 2$ [17] were significantly up-regulated in the symbiotic germinated seeds compared with free-living OMF (Table 1). Moreover, the JA biosynthesis pathway-related genes from the plant and OMF showed similar expression profiles in the symbiotic seeds, but with opposite trends. We have observed that some JA biosynthesis-related enzyme-encoding genes, such as LOX, AOS, OPR, and 3-ketoacyl-CoA thiolase (KAT) are down-regulated in the symbiotic germinated seeds relative to their expression in the asymbiotic germinated seeds, while the key genes in the Tulasnella JA biosynthesis pathway showed an up-regulated expression in the symbiotic germinated seeds. In the SL biosynthesis pathway, the isomerase-encoding gene $D 27$, two carotenoid-cleavage dioxygenases $C C D 7$ and $C C D 8$, and the CYP450 monooxygenase gene MAX1 were all up-regulated in the symbiotic germination seeds. In addition, five D14/DAD2 genes were also found to be up-regulated in the D. officinale-Tulasnella symbiotic germination seeds.

Table 1. The DEGs in the DoTc vs. Do and DoTc vs. Tc comparisons involved in the ABA (abscisic acid), JA (jasmonic acid), and SL (strigolactone) biosynthesis pathways.

\begin{tabular}{cccc}
\hline Gene Name & Gene ID & $\log 2$ (DoTc/Do) & Description \\
\hline ABA pathway & & -2.11 & Zeaxanthin epoxidase \\
\hline DoZXE-1 & Dendrobium_GLEAN_10076665 & -2.00 & Zeaxanthin epoxidase \\
DoZXE-2 & Novel00252 & -0.57 & Short-chain type dehydrogenase/reductase \\
DoSDR-1 & Dendrobium_GLEAN_10042288 & -1.33 & Short-chain type dehydrogenase/reductase \\
DoSDR-2 & Dendrobium_GLEAN_10068100 & -2.1 & Short-chain type dehydrogenase/reductase \\
DoSDR-3 & Dendrobium_GLEAN_10127951 & $\log$ 2(DoTc/Tc) & \\
\hline ABA pathway & & & Short-chain type dehydrogenase/reductase \\
& & 7.24 & dehydrogenase/reductase \\
BcABA4-1 & Cluster-2641.12008 & 2.42 & Short-chain type dehydrogenase/reductase \\
BcABA4-2 & Cluster-2152.0 & 10.43 & P450 monooxygenase protein CYP51 \\
BcABA1/2-1 & Cluster-2641.19250 & 10.01 & P450 monooxygenase protein CYP51 \\
BcABA1/2-2 & Cluster-2641.15199 & $\log 2(\mathrm{DoTc} / \mathrm{Do})$ & \\
\hline JA pathway & & -0.67 & Linoleate 13S-lipoxygenase \\
\hline DoLOX & Dendrobium_GLEAN_10090503 & -0.98 & Allene oxide synthase \\
DoAOS-1 & Dendrobium_GLEAN_10010006 & -0.40 & Allene oxide synthase \\
DoAOS-2 & Dendrobium_GLEAN_10012197 & -1.65 & 12-oxophytodienoate reductase \\
DoOPR & Dendrobium_GLEAN_10113554 & -2.50 & 3-ketoacyl-CoA thiolase \\
DoKAT & Dendrobium_GLEAN_10097185 & &
\end{tabular}


Table 1. Cont.

\begin{tabular}{cccc}
\hline Gene Name & Gene ID & & Description \\
\hline JA pathway & & $\log 2(\mathrm{DoTc} / \mathrm{Tc})$ & \\
\hline TcLOX-1 & Cluster-2641.17948 & 10.60 & Linoleate 13S-lipoxygenase \\
TcLOX-2 & Cluster-2641.19486 & 10.33 & Linoleate 13S-lipoxygenase \\
TcLOX-3 & Cluster-2641.18695 & 10.34 & Linoleate 13S-lipoxygenase \\
TcLOX-4 & Cluster-2641.17988 & 10.66 & Linoleate 13S-lipoxygenase \\
TcLOX-5 & Cluster-2641.16009 & 9.35 & Linoleate 13S-lipoxygenase \\
TcLOX-6 & Cluster-6210.0 & 6.67 & Linoleate 13S-lipoxygenase \\
TcLOX-7 & Cluster-1378.1 & 5.99 & Linoleate 13S-lipoxygenase \\
TcAOS-1 & Cluster-2641.19289 & 10.40 & Allene oxide synthase \\
TcAOS-2 & Cluster-2641.20726 & 10.07 & Allene oxide synthase \\
TcAOS-3 & Cluster-2641.16940 & 7.81 & Allene oxide synthase \\
TcAOS-4 & Cluster-2641.8055 & 6.54 & Allene oxide synthase \\
TcAOS-5 & Cluster-2641.30606 & 7.21 & Allene oxide synthase \\
\hline SL pathway & & $\log 2($ DoTc/Do) & \\
\hline DoHMGR-1 & Dendrobium_GLEAN_10079864 & 0.87 & Hydroxymethylglutaryl-CoA reductase \\
DoHMGR-2 & Dendrobium_GLEAN_10079865 & 0.91 & Hydroxymethylglutaryl-CoA reductase \\
DoD27 & Dendrobium_GLEAN_10126990 & 5.22 & B-carotene isomerase \\
DoCCD7-1 & Dendrobium_GLEAN_10008301 & 4.21 & Carotenoid cleavage dioxygenase 7 \\
DoCCD7-2 & Dendrobium_GLEAN_10009315 & 2.98 & Carotenoid cleavage dioxygenase 7 \\
DoCCD7-3 & Dendrobium_GLEAN_10049685 & 9.92 & Carotenoid cleavage dioxygenase 7 \\
DoCCD8-1 & Dendrobium_GLEAN_10070249 & 3.87 & Carotenoid cleavage dioxygenase 8 \\
DoCCD8-2 & Dendrobium_GLEAN_10072088 & 4.08 & Carotenoid cleavage dioxygenase 8 \\
DoMAX1 & Dendrobium_GLEAN_10109899 & 2.00 & Cytochrome P450 711A1 \\
DoD14-1 & Dendrobium_GLEAN_10025168 & 2.45 & Probable strigolactone esterase \\
DoD14-2 & Dendrobium_GLEAN_10114182 & 1.52 & Probable strigolactone esterase \\
DoD14-3 & Dendrobium_GLEAN_10143398 & 1.86 & Probable strigolactone esterase \\
DoDAD2-1 & Dendrobium_GLEAN_10013491 & 2.01 & Probable strigolactone esterase \\
DoDAD2-2 & Dendrobium_GLEAN_10114181 & 1.34 & Probable strigolactone esterase \\
\hline & & &
\end{tabular}

\subsection{Expression of DEGs Related to ABA, JA, and SL Hormone Biosynthesis Detected by $q R T-P C R$}

In the symbiotic and asymbiotic germinated seed samples, we successfully detected the expression of key genes related to ABA, JA, and SL biosynthesis, and the results are shown in Figure 3. Two $C C D 7$ and $C C D 8$ genes and the MAX1 gene, which are key genes in the D. officinale SL biosynthesis pathway, all showed up-regulated expression in the symbiotic germinated seed (DoTc) samples, based on expression profiles determined from transcriptome sequencing. In the symbiotic roots of $D$. officinale seedlings (SR), these genes were all down-regulated compared to the control asymbiotic root samples (ASR), which showed expression trends that were opposite to the SGS samples, with the exception of CCD8-2. In the ABA and JA biosynthesis pathways, the expression of the ZXE, SDR-1 and SDR-2, LOX, AOS-1 and AOS-2, and OPR genes from D. officinale was significantly down-regulated in the SGS and SR samples compared to one or both of the asymbiotic control samples (Do and ASR). Moreover, we also assayed the differential expression of four $B c A B A$ genes ( $B C A B A 4-1, B c A B A 4-2, B C A B A 1 / 2-1$, and $B C A B A 1 / 2-2$ ) which could be involved in ABA biosynthesis in the OMF Tulasnella sp. in the SGS and SR samples and free-living Tulasnella mycelium (Tc). We found that the expression in the SGS and SR samples was significantly up-regulated compared with that in the Tc samples, and the expression of these four genes in the SGS samples was also based on the expression trends from the transcriptome sequencing and was more significantly upregulated than in the SR samples. 

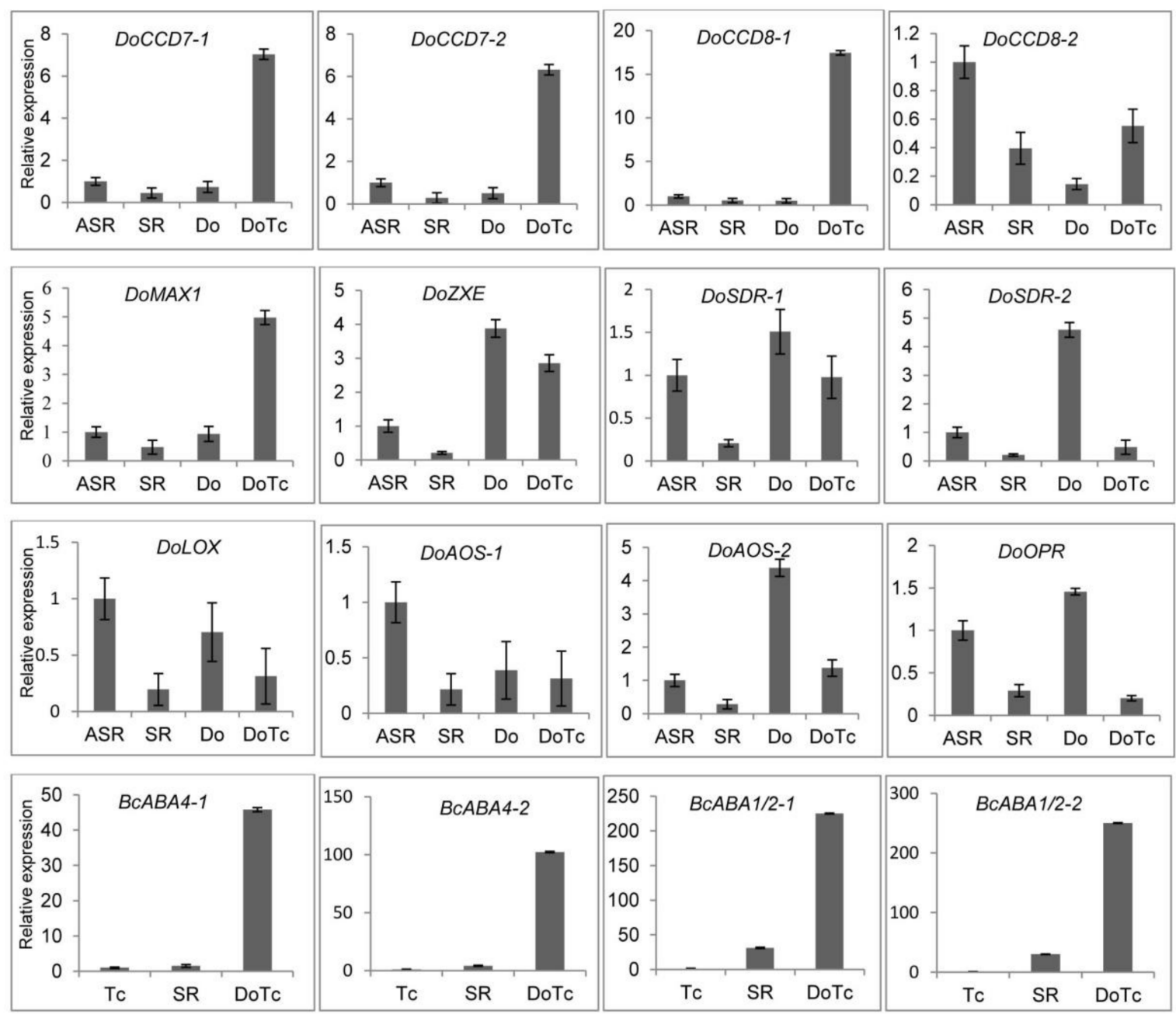

Figure 3. The expression levels of hormone biosynthesis-related genes determined by qRT-PCR. DoTc: symbiotic germinated seeds; Do: asymbiotic germinated seeds; SR: symbiotic roots of $D$. officinale seedlings; ASR: asymbiotic roots of D. officinale seedlings; Tc: free-living Tulasnella mycelium. Each data point represents the mean of three biological replicates and three experimental replicates. Bars represent the standard error of the mean, SE $(n=9)$.

\subsection{Assays of $A B A, J A$, and SL Contents}

In order to perform a deep analysis to determine their possible functions, we assayed the absolute content of ABA, JA, and 5-deoxystrigol (5-DS) in the symbiotic germinated seeds, asymbiotic germinated seeds, and free-living Tulasnella mycelium, corresponding to the RNA-Seq libraries DoTc, Do, and Tc, respectively. The results showed that in the symbiotic $D$. officinale germinated seeds, the JA concentration was slightly higher than in free-living Tulasnella mycelium and much lower than in the asymbiotic germinated seeds (Figure 4), and the ABA concentration was lower than in the two types of control samples (Figure 5). 5-DS, a precursor of SLs, is an essential index for quantifying SLs [28]. The 5-DS contents in the different tissues varied from 0.21 to $2.03 \mathrm{ng} / \mathrm{g}$. FW); the lowest content was in the free-living Tulasnella mycelium, followed by the symbiotic germinated seeds, with the highest content found in the asymbiotic germinated seeds (Figure 5).

\section{Discussion}

The role of JA signaling is well known in plant-pathogen interactions, and it also possibly results in the enhanced biosynthesis of JA in the green protocorms of Oncidium sphacelatum in association with the fungal symbiont Ceratobasidium sp. [6]. We have observed that the JA concentration in the 
symbionts is lower than in asymbiotic germinated seeds, but it is higher than in free-living fungal hyphae of Tulasnella sp. (Figure 4). Moreover, the trend of changes in JA concentration seen in the symbionts and control samples is supported by the relative expression of JA biosynthesis pathway genes. We observed that some JA biosynthesis-related enzyme-encoding genes, such as LOX, AOS, $O P R$, and $K A T$, were down-regulated in the symbiotic germinated seeds relative to their expression in the asymbiotic germinated seeds, which suggested that JA, as a kind of defense-associated plant hormone, may also be involved in the regulation of $D$. officinale seed association with mycorrhizal fungi. We can speculate that the colonization of the protocorms by the Tulasnella fungus resulted in the downgrading of JA biosynthesis-related genes, and allowed the fungus to enter cortical cells unimpeded and set up an efficient mutualism. The key genes in the Tulasnella JA biosynthesis pathway showed a significantly up-regulated expression, indicating that JA produced by Tulasnella might accumulate to a high level in the symbiotic germinated seeds.

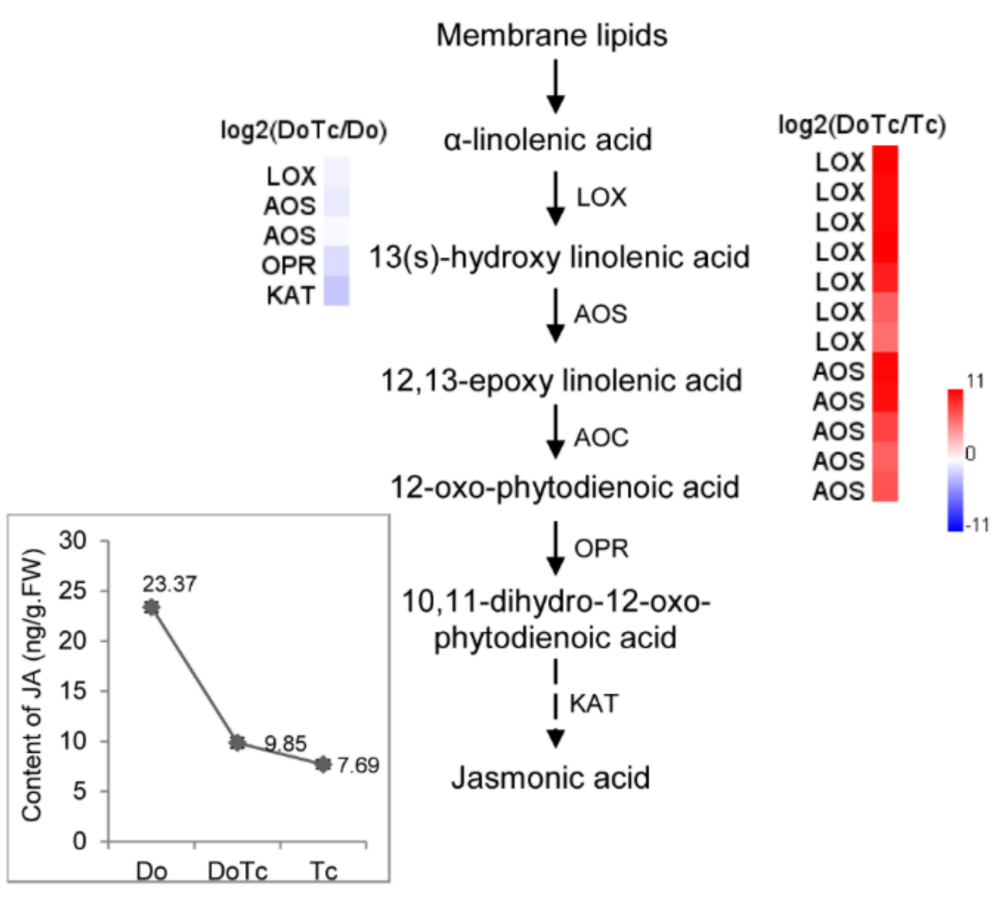

Figure 4. The genes involved in the biosynthesis of the oxylipin JA identified in the symbiotic germinated seeds of $D$. officinale in association with a Tulasnella sp. isolate. LOX: Linoleate 13S-lipoxygenase; AOS: Allene oxide synthase; AOC: Allene oxide cyclase; OPR: 12-oxophytodienoate reductase; KAT: 3-ketoacyl-CoA thiolase; JMT: Jasmonate O-methyltransferase. Red and blue indicate up- and down-regulated transcripts, respectively, from the DoTc vs. Do and DoTc vs. Tc comparisons. Each data point represents the mean of six biological replicates. Bars represent the standard error of the mean, $\mathrm{SE}(n=6)$.

The putative biosynthesis network consisting of the ABA and SL biosynthesis pathways in the symbiotic germinated seeds of the D. officinale-Tulasnella symbiosis is shown in Figure 5. In this network, the HMGR gene that encodes 3-hydroxy-3-methylglutaryl-CoA reductase, which catalyzes the reduction of 3-hydroxy-3-methylglutaryl-CoA in the mevalonate pathway, showed an induced expression in the symbiotic germinated seeds. Geranylgeranyl-PP is then catalyzed to form $\beta$-carotene, which is the critical branching point for both SL and ABA, and many of the catalyzing enzyme-related genes showed different degrees of expression in the two types of germinated seeds.

Many studies have shown a negative correlation between the concentrations of endogenous ABA and rates of seed germination, which supports the notion that high concentrations of endogenous ABA in seeds may play a critical role in preventing germination in species such as wheat (Triticum aestivum L.) [29], cacao (Theobroma cacao L.) [30], and rapeseed (Brassica rapa L.) [31]. In orchids, it 
has been suggested that high concentrations of ABA occur in seeds that are difficult to germinate such as Epipactis helleborine [32], and Calanthe tricarinata [3]. Our results showed that in symbiotic germinated D. officinale seeds, the ABA concentration was lower than in the control, indicating that low endogenous ABA levels may have positive effects on $D$. officinale symbiotic seed germination. Previous studies have shown that in symbiotic $D$. officinale seedlings, the ABA concentration was lower than in the control [33], which is in accord with the lower ABA concentration trends observed in the symbiotic germinated seeds compared to the asymbiotic germinated seeds. In our study, the expression of SDR and ZXE, two genes in the ABA biosynthesis pathway, was significantly suppressed in the symbiotic germinated seeds. These results agree with those of a recent study in which high concentrations of exogenously applied ABA were also found to suppress seed germination in vitro [3].

In our study, the relative transcription of most of the key MVA pathway genes from Tulasnella was significantly up-regulated, as was the transcription of some critical ABA biosynthesis unigenes with homologs in Botrytis; examples are two putative BcABA4 unigenes (Cluster-2641.12008 with 7.24-fold up-regulation and Cluster-2152.0 with 2.42-fold up-regulation) and two putative $\mathrm{P} 450$ monooxygenase protein CYP51 genes (BCABA1 or BCABA2; Cluster-2641.19250 with 10.43-fold up-regulation and Cluster-2641.15199 with 10.01-fold up-regulation) in the symbiotic germinated seeds compared to the asymbiotic fungus Tulasnella sp. (Figure 5 and Table 1), indicating that symbiotic Tulasnella sp. might produce more activated ABA than the control. These results are consistent with previous reports showing that $\mathrm{ABA}$ affects mycelium growth [11]. In Ceratocystis fimbriata, the exogenous application of ABA increased fungal growth slightly. In Magnaporthe oryzae, ABA increased spore germination and the formation of appressoria, specialized infection structures for breaking down the plant cell wall and allowing invasion [11]. Moreover, the Tulasnella sp. ABA-INSENSITIVE-like protein (Cluster-2641.20009 with 8.94-fold up-regulation) and five ABA receptor PYL-related unigenes (6.54- to 10.06-fold up-regulation) were also found to be significantly up-regulated in the symbiotic germinated seeds compared with the asymbiotic Tulasnella sp. fungus. There is also emerging evidence to suggest that ABA plays an important role in compatible interactions in mutualistic plant-microbe interactions [18], and it can promote the infection and establishment of compatible interactions with arbuscular mycorrhizal fungi [16]. However, we found that the ABA concentration in the orchid symbionts is also lower than in the free-living fungal mycelium of Tulasnella sp. Based on these results, we can hypothesize that the symbiotic fungus Tulasnella produces more activated ABA to promote the recognition and establishment of compatible interactions with its mutualist orchid partner in early seed germination (stages 1-3); it is during these early stages that an abundance of pelotons is usually formed. After protocorm formation, the germinated seed develops to the fourth stage (emergence of leaf from shoot region), the reinvaded hyphae conspicuously decrease, and the hyphae are degraded in the symbiotic orchid cell [34]. Thus, there might be both a collapsed hyphae and healthy pelotons that can be observed. We have detected one putative abscisic acid $8^{\prime}$-hydroxylase-encoding gene, CYP707A (Cluster-3911.0), from Tulasnella that is highly expressed in symbiotic germinated seeds but not in asymbiotic Tulasnella, and this enzyme can degrade and deplete endogenous ABA levels to regulate intracellular ABA homeostasis in plants [16,35]. In line with its important role as a phytohormone, ABA concentrations in the plant are controlled by a tightly regulated balance between biosynthesis, inactivation, and degradation [36]. At this stage, low concentrations of ABA in the symbiont could promote $D$. officinale seed germination and inhibit the growth of Tulasnella mycelia.

In our study, we also detected a precursor of SLs (5-DS) in the germinated seeds of $D$. officinale, and in addition, we found that genes encoding key enzymes involved in the conversion of $\beta$-carotene to carlactone and mature SLs, D27, CCD7, CCD8, and MAX1 are expressed in both types of germination seeds, indicating that SLs might also play important roles in regulating $D$. officinale seed germination. Moreover, the content of 5-DS in the asymbiotic germinated seeds $(2.03 \mathrm{ng} / \mathrm{g} \cdot \mathrm{FW})$ was much higher than in the symbiotic germinated seeds $(0.81 \mathrm{ng} / \mathrm{g} \cdot \mathrm{FW})$, and this result was supported by the slow growth rate of the asymbiotic germinated seeds compared with that of the symbiotic germinated seeds, indicating that high concentrations of SLs or their biosynthetic precursors might inhibit growth. 
In other plants, studies have shown that SLs or their biosynthetic precursors act as plant hormones that inhibit shoot branch growth [22-25]. In addition, the expression of the SL biosynthesis and signal pathway genes increased significantly ( $>2$-fold) in the symbiotic germinated seeds compared to the asymbiotic germinated seeds, which possibly have enhanced the branching of the Tulasnella fungus within orchid tissues to maximize the number of pelotons formed. This model might be supported by previous studies showing that SLs also promote branched growth in AM fungi [22,23]. Nevertheless, based on the lower level of 5-DS in the symbiotic germinated seeds and the higher level of 5-DS in the asymbiotic germinated seeds, we can speculate that the symbiotic fungus Tulasnella might suppress the biosynthesis of orchid SLs in order to maintain a low concentration of SLs to accelerate the germination rate in the D. officinale-Tulasnella symbiosis. Additionally, the germinated seed developed to the fourth stage (emergence of the leaf from the shoot region), the reinvaded hyphae conspicuously decreased, and the hyphae degraded in the symbiotic orchid cell [34]. At this stage, a low concentration of SLs in the symbiont might also slow the growth of the reinvaded Tulasnella mycelium.

Previous studies have suggested that ABA is one of the regulators of the strigolactone biosynthesis that acts through an as-yet-unknown mechanism [36]. Moreover, Toh et al. have proposed that host-exudate SLs reduce ABA levels to alleviate seed thermoinhibition in Arabidopsis [37,38]. This suggests that there could be complicated cross-talk among the hormones in the orchid-fungus symbionts that warrant future study.

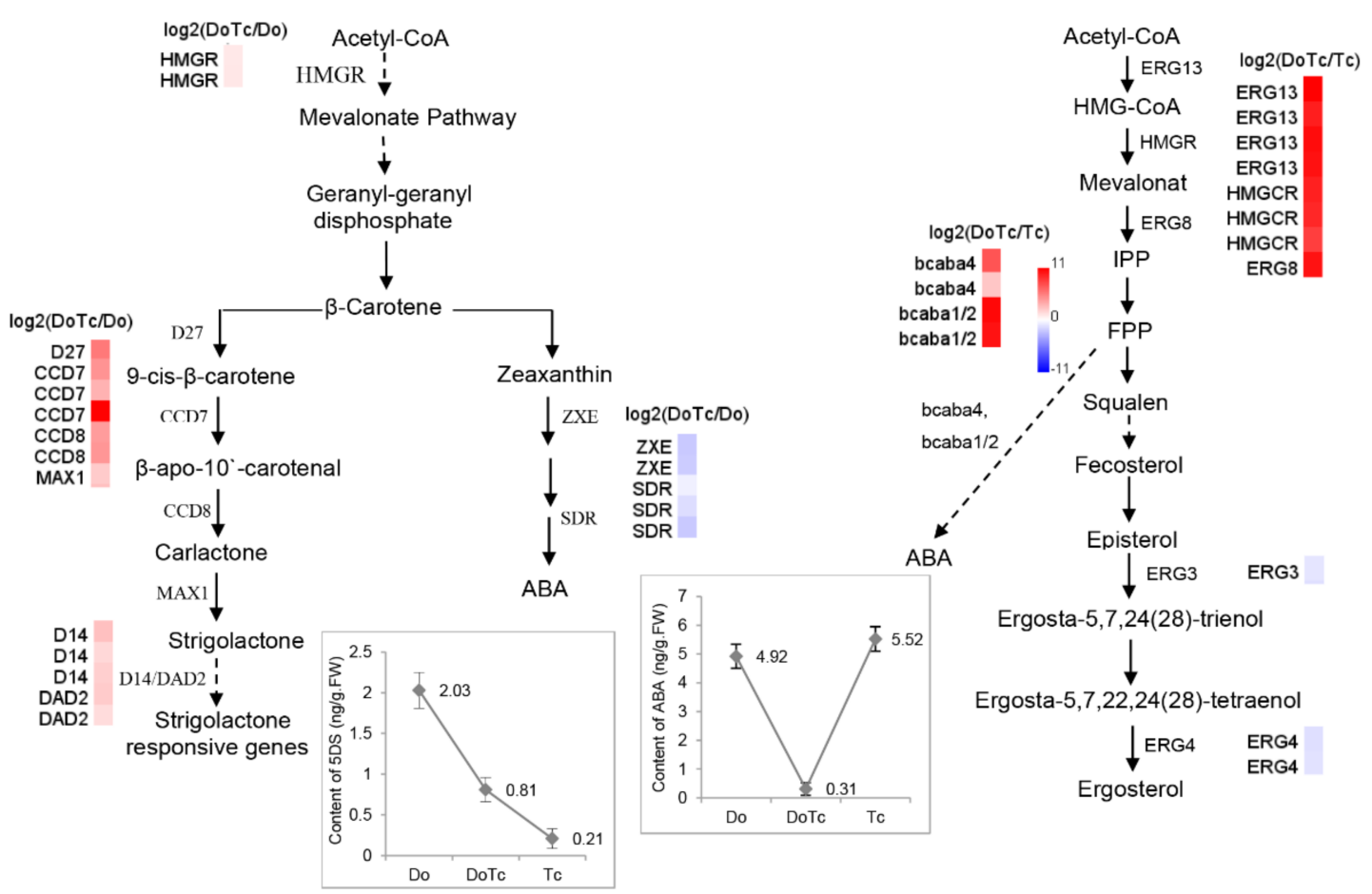

Figure 5. The diagram showing the proposed stigmasterol, ABA, and ergosterol biosynthesis pathways in the symbiotic germinated seeds of $D$. officinale in the symbiotic association with a Tulasnella sp. isolate. HMGR: Hydroxymethylglutaryl-CoA reductase; D27: $\beta$-carotene isomerase; CCD: carotenoid cleavage dioxygenase; MAX: more axillary growth; ZXE: zeaxanthin epoxidase, SDR: short-chain dehydrogenase/reductase; HMG: Hydroxymethylglutaryl; ERG13/HMGS: Hydroxymethylglutaryl-CoA synthase; ERG8: phosphomevalonate kinase; FPP: Farnesyl-Pyrophosphate; ERG4: sterol C-24 reductase. Red and blue indicate up- and down-regulated transcripts, respectively, from the DoTc vs. Do and DoTc vs. Tc comparisons. Each data point represents the mean of six biological replicates. Bars represent the standard error of the mean, SE $(n=6)$. The solid arrows represent direct relationships, and the dashed arrows represent indirect relationships. 


\section{Materials and Methods}

\subsection{Symbiotic and Asymbiotic Germination of D. officinale Seeds}

D. officinale plants were grown in the greenhouse of the Chinese Academy of Forestry (Beijing, China) at day / night temperatures of $24{ }^{\circ} \mathrm{C} / 18{ }^{\circ} \mathrm{C}$, a relative air humidity of $65-75 \%$, a 10-h day /14-h night photoperiod, and an irradiance of $700 \mathrm{~mol} \mathrm{~m}^{-2} \cdot \mathrm{s}^{-1}$. Six D. officinale seed capsules were collected from the greenhouse and divided into two groups after surface sterilization. Seeds from the first group were cultured with Tulasnella sp., which was isolated from Cymbidium goeringii roots (collected from Suizhou, China); a BLAST search showed that the ITS sequences from this isolate were $99 \%$ homologous to the ITS from T. calospora isolate Pv-PC-2-1 (NCBI accession No: GU166418.1) [39]. The seeds with Tulasnella sp. were cultured on $8 \mathrm{~g} / \mathrm{L}$ oatmeal agar (OMA) medium; this group was defined as DoTc. The second group of seeds was cultured on the same OMA medium without fungi. Seeds were sown and incubated according to the procedures described by Xu et al. [39]. The DoTc seeds germinated and developed to the fourth stage (appearance of the first true leaf, germination rate around at 90\%) [12,34], at which point they were cleared in $\mathrm{KOH}$ and stained using Trypan blue [39,40]. Microscopic observation showed that the intracellular hyphae stained blue in the DoTc germinated seeds, which indicated that a symbiotic system was established between D. officinale and Tulasnella sp. The group of seeds germinated without fungi had a very low germination rate $(0.1 \%)$, and we did not find fungal hyphae; these were defined as asymbiotic germinated seeds (Do). Free-living fungal mycelium was maintained on an OMA medium at $25^{\circ} \mathrm{C}$ for 10 days (Tc). DoTc, Do, and Tc (three biological replicates) were collected, immediately frozen in liquid nitrogen, and stored at $-80{ }^{\circ} \mathrm{C}$ prior to RNA extraction.

\section{2. cDNA Library Construction and Nucleotide Sequencing}

The total RNA was extracted from each sample using the RNeasy Plant Mini Kit (Qiagen, Hilden, Germany) and treated with an RNase-free DNase I digestion kit (Aidlab, Beijing, China) to remove residual genomic DNA. RNA degradation and contamination was monitored by electrophoresis on $1 \%$ agarose gels. RNA purity was checked using the NanoPhotometer ${ }^{\circledR}$ spectrophotometer (Implen, Westlake Vilagge, CA, USA). RNA concentration was measured with the Qubit ${ }^{\circledR}$ RNA Assay Kit in the Qubit $^{\circledR}$ 2.0 Fluorometer (Life Technologies, Carlsbad, CA, USA). RNA integrity was assessed using the RNA Nano 6000 Assay Kit on the Bioanalyzer 2100 system (Agilent Technologies, Santa Clara, CA, USA).

For each sample, $3 \mu \mathrm{g}$ of the total RNA was used as input material for the RNA-Seq library preparations. Sequencing libraries were generated using the NEBNext ${ }^{\circledR}$ Ultra $^{\text {TM }}$ RNA Library Prep Kit for Illumina ${ }^{\circledR}$ (NEB, Ipswitch, MA, USA) following the manufacturer's recommendations, and index codes were added to attribute the sequencing reads to each sample. The PCR products were purified (AMPure XP system) and the library quality was assessed on the Agilent Bioanalyzer 2100. The RNA-Seq libraries were sequenced on an Illumina Hiseq 4000 instrument to generate $150 \mathrm{bp}$ paired-end reads. All samples were sequenced with three biological replicates.

\subsection{Transcriptome Data Analysis}

\subsubsection{Quality Control and cDNA Assembly}

Raw reads in the FASTQ format were first processed using in-house custom Perl scripts. In this step, clean reads were obtained by removing reads containing adapters, poly- $N$-containing reads, and low quality reads from the raw data. Additionally, the Q20 and Q30 scores, and the GC content of the clean reads were calculated simultaneously. All downstream analyses were based on high-quality clean data.

Sequence read mapping to the D. officinale Reference Genome: The index of the D. officinale reference genome [27] was built using Bowtie v2.2.3, and the paired-end reads were aligned to the 
reference genome using TopHat v2.0.12 with mismatch set to 2 and the default values used for the other parameters. The OMF transcriptome de novo assembly was accomplished using Trinity [41] with the min_kmer_cov set to 2 by default and all other parameters set to the default values. The coding sequences were predicted using Estscan v3.0.3 with the default settings [42]. All assembled unigene sequences in this study are given in Table S5.

\subsubsection{Quantification of Gene Expression Levels}

HTSeq v0.6.1 was used to count the read numbers mapped to each D. officinale gene. The FPKM (expected number of Fragments Per Kilobase of transcript sequence per Million base pairs sequenced) was then calculated for each gene based on the length of the gene and the read count mapped to the gene [43].

Gene expression levels in the OMF were estimated using RSEM with the default settings [44]. The clean reads were mapped to the reference sequences which were assembled by Trinity [41] using the clean read data from the Tc library, and the transcript read counts were obtained and converted to FPKM for gene expression level analysis in the OMF [43].

\subsubsection{Differential Gene Expression Analysis}

A differential expression analysis of the two conditions/groups (two biological replicates per condition) was performed using the DESeq R package (1.18.0) with the default settings [45]. DESeq provides statistical routines for determining differential expression in digital gene expression data using a model based on the negative binomial distribution. The resulting $p$-values were adjusted using the Benjamini-Hochberg procedure for controlling the false discovery rate. Genes with a $\log _{2}$ (Fold_Change) $>1$ and adjusted $p$-value $<0.001$ identified by DESeq were considered to be differentially expressed.

\subsubsection{Unigene Functional Annotation and Metabolic Pathway Analysis}

Unigene function was annotated based on the seven databases reported by Liu et al. [46]. Metabolic pathway analysis was performed using the Kyoto Encyclopedia of Genes and Genomes Pathway database (KEGG: http:/ / www.genome.jp/kegg/). We used the KOBAS [47] software to test the statistical enrichment of DEGs in the KEGG pathways.

\subsection{Hormone Extraction, Fractionation, and UPLC-ESI-qMS/MS Analysis}

The concentrations of the ABA, JA, and SL hormones in the Do, DoTc, and Tc samples were measured using ultraperformance liquid chromatography coupled with a tandem quadrupole mass spectrometer equipped with an electrospray interface (UPLC-ESI-qMS/MS). Six biological replicates were used in the hormone quantification measurements.

1. 5-DS: To isolate 5-DS, $1 \mathrm{~g}$ (fresh weight) samples of symbiotic or asymbiotic seeds were frozen in liquid nitrogen, ground to a powder, transferred to $2 \mathrm{~mL}$ microcentrifuge tubes, and then extracted with $10 \mathrm{~mL}$ acetone with ultrasound in a water bath for $15 \mathrm{~min}$, after which the tubes were kept at $-20^{\circ} \mathrm{C}$ for at least $12 \mathrm{~h}$. After centrifugation at $8000 \mathrm{rpm}$ for $5 \mathrm{~min}$, the supernatants were transferred into new tubes and centrifuged at $13,000 \mathrm{rpm}$ at $4{ }^{\circ} \mathrm{C}$ for $5 \mathrm{~min}$. The lower organic phases were then transferred to new tubes and evaporated to dryness in a stream of dry $\mathrm{N}_{2}$. The crude extracts were dissolved in $3 \mathrm{~mL}$ normal hexane and filtered through spin columns (500 mg, $6 \mathrm{~mL}$, Agilent Technologies Corporation, Santa Clara, CA, USA) with $5 \mathrm{~mL}$ of normal hexane, and the elution products were then evaporated to dryness in a stream of $\mathrm{N}_{2}$. The crude extracts were then dissolved in $200 \mu \mathrm{L}$ of methanol and filtered through spin columns (Ultra-Free MC, $0.22 \mu \mathrm{m}$ pore size; Millipore, Tokyo, Japan), and subjected to an HPLC-MS/MS analysis.

Identification of 5-DS by LC-MS/MS: HPLC separation was conducted with an Agilent 1290 HPLC instrument (Agilent Technologies Corporation) fitted with a poroshell 120 SB-C18 column $(2.1 \mathrm{~mm} \times 150 \mathrm{~mm}, 2.7 \mu \mathrm{m})$, and $2 \mu \mathrm{L}$ was injected for the HPLC-MS/MS analysis. The column 
temperature was kept at $30^{\circ} \mathrm{C}$. The binary mobile phase system consisted of methanol (A) and water (B) in $0.1 \%$ formic acid under a gradient condition $(0-0.5$ and $5.1-8.00 \mathrm{~min}, 80 \% \mathrm{~A}, 3-5 \mathrm{~min}, 90 \%$ B) with a flow rate of $0.3 \mathrm{~mL} / \mathrm{min}$. Mass spectrometry was performed with a SCIEX-6500Qtrap (MS/MS) mass spectrometer (AB SCIEX LLC., Framingham, MA, USA) equipped with an electrospray source. The drying and nebulizing gas was nitrogen generated from pressurized air in an N2G nitrogen generator (MIU instruments, Hangzhou, China). The curtain gas was set to $25 \mathrm{psi}$, the nebulizer gas was set to $55 \mathrm{psi}$, the heater gas was set to $65 \mathrm{psi}$, and the ion spray voltage was set to $5000 \mathrm{~V}$. The atomization temperature was set to $350{ }^{\circ} \mathrm{C}$. Analytes were quantified in the multiple-reaction-monitoring (MRM) mode for the known 5-DS detection, at $m / z$ 331.0/234.1 with a declustering potential (DP) of $45 \mathrm{~V}$ and collision energy (CE) of $14 \mathrm{~V}$.

2. ABA and JA: To isolate the hormones, $1 \mathrm{~g}$ (fresh weight) samples of symbiotic or asymbiotic seeds were frozen in liquid nitrogen, ground to a powder, and transferred to $2 \mathrm{~mL}$ microcentrifuge tubes. The ground tissue was extracted in $10 \mathrm{~mL}$ isopropanol/hydrochloric acid buffer by shaking at $4{ }^{\circ} \mathrm{C}$ for $30 \mathrm{~min}$, and $20 \mathrm{~mL}$ of dichloromethane was then added and the mixture was vibrated at $4{ }^{\circ} \mathrm{C}$ for a further $30 \mathrm{~min}$. The tubes were centrifuged at $13,000 \mathrm{rpm}$ at $4{ }^{\circ} \mathrm{C}$ for $5 \mathrm{~min}$, and the lower organic phase was transferred to a new tube and was then evaporated to dryness in a stream of $\mathrm{N}_{2}$. The extracts were dissolved in $400 \mu \mathrm{L}$ of methanol and filtered through spin columns (Ultra-Free MC, $0.22 \mu \mathrm{m}$ pore size; Millipore, Tokyo, Japan), and subjected to an HPLC-MS/MS analysis.

ABA and JA were measured with an HPLC-ESI-qMS/MS instrument (AQUITY UPLC ${ }^{\text {TM }}$ System/Quattro Ultima Pt; Waters, Milford, MA, USA) fitted with an AQUITY UPLC BEH C18 column $(1.7 \mu \mathrm{m}, 2.1 \times 100 \mathrm{~mm}$, Waters, Milford, MA, USA). The column temperature was kept at $40{ }^{\circ} \mathrm{C}$. A binary mobile phase system consisting of $2 \%(v / v)$ methanol, $0.05 \%$ formic acid, and $5 \mathrm{mmol} / \mathrm{L}$ ammonium acetate in water (A) and acetonitrile (B) under a gradient condition $(0-0.25$ and $5.01-6.00 \mathrm{~min}, 90 \%(\mathrm{~A}), 4-5 \mathrm{~min}, 100 \%$ (B)) with a flow rate of $0.3 \mathrm{~mL} / \mathrm{min}$. The sample chamber temperature was set to $10^{\circ} \mathrm{C}$ and the injection volume was $10 \mu \mathrm{L}$. The capillary voltage was controlled at $20 \mathrm{~V}$. The ion source temperature was set to $150{ }^{\circ} \mathrm{C}$, the cone gas flow was $50 \mathrm{~L} / \mathrm{h}$, desolvation temperature was $400{ }^{\circ} \mathrm{C}$, and the desolvation gas flow was $800 \mathrm{~L} / \mathrm{h}$. Analytes were also quantified in multiple-reaction-monitoring (MRM) mode for the known ABA and JA detections.

For ABA quantification, the declustering potential was $-25 \mathrm{~V}$, the collision energy $-25 \mathrm{~V}$, and the ion transition was at $m / z 263.2 / 204.0$; for JA quantification, the declustering potential was $-32 \mathrm{~V}$, the collision energy $-12 \mathrm{~V}$, and the ion transition was at $m / z 59.0 / 109.0$.

\subsection{Differential Gene Expression Detected by qRT-PCR}

The symbiotic and asymbiotic root samples from the D. officinale seedlings $(10 \mathrm{~cm}$ in height $)$ that were previously used to prepare the RNA-Seq libraries were used to perform qRT-PCR assays for gene expression quantification. The plant materials were flash-frozen in liquid nitrogen and stored at $-80{ }^{\circ} \mathrm{C}$ prior to RNA extraction. RNA isolation, qRT-PCR, and calculations of relative mRNA levels were performed as previously described [48]. Each data point represents the mean of three biological replicates and three experimental replicates. DoEF1 $\alpha$ and DoRPL30 [49] from D. officinale, and TcEF1 $\alpha$ [50] and TcTUBB (Cluster-2641.19171, Table S5) from Tulasnella were used as reference genes for the normalization of gene expression, respectively. The primer sequences are shown in Table S9, and their amplification efficiencies were within the range of $95-105 \%$.

\section{Conclusions}

In this study, we generated transcriptome datasets of $D$. officinale germinated seeds to examine the expression profiles of genes related to the biosynthesis of three types of endogenous hormones-JA, ABA, and SLs-in the D. officinale-Tulasnella symbionts, asymbiotic germinated seeds, and the free-living OMF. We also performed comprehensive analyses of the contents of individual hormones, and assayed gene expression using qRT-PCR in the symbiotic and asymbiotic seed samples. The results allowed us to propose potential regulatory mechanisms for JA, ABA, and SLs from the orchid and 
OMF in the D. officinale-Tulasnella symbionts. Our study will provide new insights into the molecular mechanisms that regulate the orchid-fungus symbiosis.

Supplementary Materials: The following are available online at http:/ / www.mdpi.com/1422-0067/19/11/3484/ s1.

Author Contributions: T.W. designed the experiments, analyzed the data and wrote the paper; Z.S., X.W., and L.X. performed the experiments; L.L. conceived, led, and supervised the study. Q.S. and L.L. were responsible for this work.

Funding: This work was supported by the Beijing Science and Technology Projects of China [grant number Z171100001417006]. The transcriptome data have been deposited at the National Center for Biotechnology Information Sequence Read Archive under accession number SRP136098.

Conflicts of Interest: The authors declare no conflicts of interest.

\section{References}

1. Arditti, J. Factors affecting the germination of orchid seeds. Bot. Rev. 1967, 33, 1-97. [CrossRef]

2. Da Silva, T.J.A.; Tsavkelova, E.A.; Zeng, S.; Ng, T.B.; Parthibhan, S.; Dobranszki, J.; Cardoso, J.C.; Rao, M.V. Symbiotic in vitro seed propagation of Dendrobium: Fungal and bacterial partners and their influence on plant growth and development. Planta 2015, 242, 1-22. [CrossRef] [PubMed]

3. Lee, Y.; Lu, C.; Chung, M.; Yeung, E.C.; Lee, N. Developmental changes in endogenous abscisic acid concentrations and asymbiotic seed germination of a terrestrial orchid, Calanthe tricarinata Lindl. J. Am. Soc. Hortc. Sci. 2007, 132, 246-252.

4. Liu, H.; Luo, Y.; Liu, H. Studies of mycorrhizal fungi of Chinese orchids and their role in orchid conservation in China: A review. Bot. Rev. 2010, 76, 241-262. [CrossRef]

5. Zhao, M.; Zhang, G.; Zhang, D.; Hsiao, Y.; Guo, S. ESTs analysis reveals putative genes involved in symbiotic seed germination in Dendrobium officinale. PLoS ONE 2013, 8, e72705. [CrossRef] [PubMed]

6. Valadares, R.B.S.; Perotto, S.; Santos, E.C.; Lambais, M.R. Proteome changes in Oncidium sphacelatum (Orchidaceae) at different trophic stages of symbiotic germination. Mycorrhiza 2014, 24, 349-360. [CrossRef] [PubMed]

7. López-Chávez, M.Y.; Guillén-Navarro, K.; Bertolini, V.; Encarnación, S.; Hernández-Ortiz, M.; Sánchez-Moreno, I.; Damon, A. Proteomic and morphometric study of the in vitro interaction between Oncidium sphacelatum Lindl. (Orchidaceae) and Thanatephorus sp. RG26 (Ceratobasidiaceae). Mycorrhiza 2016, 26, 353-365. [CrossRef] [PubMed]

8. Miura, C.; Yamaguchi, K.; Miyahara, R.; Yamamoto, T.; Fuji, M.; Yagame, T.; Imaizumi-Anraku, H.; Yamato, M.; Shigenobu, S.; Kaminaka, H. The mycoheterotrophic symbiosis between orchids and mycorrhizal fungi possesses major components shared with mutualistic plant-mycorrhizal symbioses. Mol. Plant Microbe Interact. 2018. [CrossRef] [PubMed]

9. Chen, Y.W.; Wu, J.R.; Duan, X.T.; Qin, H.X. Plant hormones produced from symbionts of Cymbidium goeringii and four Rhizoctonia-like strains. Chin. Guizhou Agric. Sci. 2017, 45, 84-86. [CrossRef]

10. Chen, J.; Liu, S.S.; Kohler, A.; Yan, B.; Luo, H.M.; Chen, X.M.; Guo, S.X. iTRAQ and RNA-seq analyses provide new insights into regulation mechanism of symbiotic germination of Dendrobium officinale seeds (Orchidaceae). J. Proteome Res. 2017, 16, 2174-2187. [CrossRef] [PubMed]

11. Chanclud, E.; Morel, J. Plant hormones: A fungal point of view. Mol. Plant Pathol. 2016, 17, $1289-1297$. [CrossRef] [PubMed]

12. Stewart, S.L.; Zettler, L.W. Symbiotic germination of three semiaquatic rein orchids (Habenaria repens, H. quinquiseta, H. macroceratitis) from florida. Aquatic Bot. 2002, 72, 25-35. [CrossRef]

13. Nambara, E.; Marion-Poll, A. Abscisic acid biosynthesis and catabolism. Ann. Rev. Plant Biol. 2005, 56, 165-185. [CrossRef] [PubMed]

14. Oritani, T.; Kiyota, H. Biosynthesis and metabolism of abscisic acid and related compounds. Nat. Prod. Rep. 2003, 20, 414-425. [CrossRef] [PubMed]

15. Gong, T.; Shu, D.; Yang, J.; Ding, Z.T.; Tan, H. Sequencing and transcriptional analysis of the biosynthesis gene cluster of abscisic acid-producing Botrytis cinerea. Int. J. Mol. Sci. 2014, 15, 17396-17410. [CrossRef] [PubMed] 
16. Lievens, L.; Pollier, J.; Goossens, A.; Beyaert, R.; Staal, J. Abscisic acid as pathogen effector and immune regulator. Front. Plant Sci. 2017, 8, 587. [CrossRef] [PubMed]

17. Siewers, V.; Smedsgaard, J.; Tudzynski, P. The P450 monooxygenase BcABA1 is essential for abscisic acid biosynthesis in Botrytis cinerea. Appl. Environ. Microb. 2004, 70, 3868-3876. [CrossRef] [PubMed]

18. Stec, N.; Banasiak, J.; Jasiński, M. Abscisic acid: An overlooked player in plant-microbe symbioses formation. Acta Biochim. Pol. 2016, 63, 53-58. [CrossRef] [PubMed]

19. Hirai, N.; Yoshida, R.; Todoroki, Y.; Ohigashi, H. Biosynthesis of abscisic acid by the non-mevalonate pathway in plants, and by the mevalonate pathway in fungi. Biosci. Biotech. Biochem. 2000, 64, 1448-1458. [CrossRef] [PubMed]

20. Ding, Z.; Zhang, Z.; Zhong, J.; Di, L.; Zhou, J.; Yang, J.; Xiao, L.; Shu, D.; Tan, H. Comparative transcriptome analysis between an evolved abscisic acid-overproducing mutant Botrytis cinerea TBC-A and its ancestral strain Botrytis cinerea TBC-6. Sci. Rep. 2016, 6, 37487-37499. [CrossRef] [PubMed]

21. Zdyb, A.; Salgado, M.G.; Demchenko, K.N.; Brenner, W.G.; Płaszczyca, M.; Stumpe, M.; Herrfurth, C.; Feussner, I.; Pawlowski, K. Allene oxide synthase, allene oxide cyclase and jasmonic acid levels in Lotus japonicus nodules. PLoS ONE 2018, 13, 1-16. [CrossRef] [PubMed]

22. Liao, D.; Liu, J.; Liu, J.; Yang, X.; Chen, X.; Gu, M.; Gu, A. Advances in the response and modulation of phytohormones on arbuscular mycorrhizal symbiosis. J. Plant Nutr. Fert. 2016, 22, 1679-1689. [CrossRef]

23. Prandi, C.; Cardinale, F. Strigolactones: A New Class of Plant Hormones with Multifaceted Roles; John Wiley \& Sons: Chichester, UK, 2014. [CrossRef]

24. Umehara, M.; Hanada, A.; Yoshida, S.; Akiyama, K.; Arite, T.; Takeda-Kamiya, N.; Magome, H.; Kamiya, Y.; Shirasu, K.; Yoneyama, K.; et al. Inhibition of shoot branching by new terpenoid plant hormones. Nature 2008, 455, 195-200. [CrossRef] [PubMed]

25. Umehara, M.; Cao, M.; Akiyama, K.; Akatsu, T.; Seto, Y.; Hanada, A.; Li, W.; Takeda-Kamiya, N.; Morimoto, Y.; Yamaguchi, S. Structural requirements of strigolactones for shoot branching inhibition in rice and Arabidopsis. Plant Cell Physiol. 2015, 56, 1059-1072. [CrossRef] [PubMed]

26. Saeed, W.; Naseem, S.; Ali, Z. Strigolactones biosynthesis and their role in abiotic stress resilience in plants: A critical review. Front. Plant Sci. 2017, 8, 1487-1500. [CrossRef] [PubMed]

27. Yan, L.; Wang, X.; Liu, H.; Tian, Y.; Lian, J.; Yang, R.; Hao, S.; Wang, X.; Yang, S.; Li, Q.; et al. The genome of Dendrobium officinale illuminates the biology of the important traditional Chinese orchid herb. Mol. Plant 2015, 8, 922-934. [CrossRef] [PubMed]

28. Liu, Y.; Li, G.; Gui, R.; Zhang, H.; Hu, X. Determination of strigolactones extracted from root of Phyllostachys edulis by ultra performance liquid chromatography. J. Zhejiang Forest. Coll. 2013, 30, 607-610.

29. Walker-Simmons, M. ABA levels and sensitivity in developing wheat embryos of sprouting resistant and susceptible cultivars. Plant Physiol. 1987, 84, 61-66. [CrossRef] [PubMed]

30. Pence, V.C. Abscisic acid and the maturation of cacao embryos in vitro. Plant Physiol. 1992, 98, $1391-1395$. [CrossRef] [PubMed]

31. Finkelstein, R.R.; Crouch, M.L. Rapeseed embryo development in culture on high osmoticum is similar to that in seeds. Plant Physiol. 1986, 81, 907-912. [CrossRef] [PubMed]

32. Van, K.G. Abscisic acid in terrestrial orchid seeds: A possible impact on their germination. Lindleyana 1987, 2, 84-87.

33. Wu, H.; Song, X.; Yang, S.; Wang, J. Effects of symbiotic fungi on the physiological characteristics of Dendrobium catenatum Lindley (Orchidaceae). Chin. Plant Sci. J. 2011, 29, 738-742. [CrossRef]

34. Chen, J.; Wang, H.; Liu, S.S.; Li, Y.Y.; Guo, S.X. Ultrastructure of symbiotic germination of the orchid Dendrobium officinale with its mycobiont, Sebacina sp. Aust. J. Bot. 2014, 62, 229-234. [CrossRef]

35. Umezawa, T.; Okamoto, M.; Kushiro, T.; Nambara, E.; Oono, Y.; Kobayashi, M.S.M.; Koshiba, T.; Kamiya, Y.; Shinozaki, K. CYP707A3, a major ABA 8'-hydroxylase involved in dehydration and rehydration response in Arabidopsis thaliana. Plant J. 2006, 46, 171-182. [CrossRef] [PubMed]

36. Zeevaart, J.A.D.; Creelman, R.A. Metabolism and physiology of abscisic acid. Annu. Rev. Plant Biol. 1988, 39, 439-473. [CrossRef]

37. Lopez-Raez, J.A.; Kohlen, W.; Charnikhova, T.; Mulder, P.; Undas, A.K.; Sergeant, M.J.; Verstappen, F.; Bugg, T.D.; Thompson, A.J.; Ruyter-Spira, C.; et al. Does abscisic acid affect strigolactone biosynthesis? New Phytol. 2010, 187, 343-354. [CrossRef] [PubMed] 
38. Toh, S.; Kamiya, Y.; Kawakami, N.; Nambara, E.; McCourt, P.; Tsuchiya, Y. Thermoinhibition uncovers a role for strigolactones in Arabidopsis seed germination. Plant Cell Physiol. 2012, 53, 107-117. [CrossRef] [PubMed]

39. Xu, L.; Tian, J.; Wang, T.; Li, L. Symbiosis established between orchid and Tulasnella spp. fungi. J. Nucl. Agric. Sci. 2017, 31, 876-883. [CrossRef]

40. Zhao, X.; Yang, J.; Liu, S.; Chen, C.; Zhu, H.; Cao, J. The colonization patterns of different fungi on roots of Cymbidium hybridum plantlets and their respective inoculation effects on growth and nutrient uptake of orchid plantlets. World J. Microbiol. Biotech. 2014, 30, 1993-2003. [CrossRef] [PubMed]

41. Grabherr, M.G.; Haas, B.J.; Yassour, M.; Levin, J.Z.; Thompson, D.A.; Amit, I.; Xian, A.L.F.; Raychowdhury, R.; Zeng, Q.; Chen, Z.; et al. Full-length transcriptome assembly from RNA-Seq data without a reference genome. Nat. Biotechnol. 2011, 29, 644-652. [CrossRef] [PubMed]

42. Haas, B.J.; Papanicolaou, A.; Yassour, M.; Grabherr, M.; Blood, P.D.; Bowden, J.; Couger, M.B.; Eccles, D.; Li, B.; Lieber, M.; et al. De novo transcript sequence reconstruction from RNA-seq using the Trinity platform for reference generation and analysis. Nat. Protoc. 2013, 8, 1494-1512. [CrossRef] [PubMed]

43. Trapnell, C.; Williams, B.A.; Pertea, G.; Mortazavi, A.; Kwan, G.; van Baren, M.J.; Salzberg, S.L.; Wold, B.J.; Pachter, L. Transcript assembly and quantification by RNA-seq reveals unannotated transcripts and isoform switching during cell differentiation. Nat. Biotechnol. 2010, 28, 511-515. [CrossRef] [PubMed]

44. Li, B.; Dewey, C.N. RSEM: Accurate transcript quantification from RNA-Seq data with or without a reference genome. BMC Bioinform. 2011, 12, 323-339. [CrossRef] [PubMed]

45. Wang, L.; Feng, Z.; Wang, X.; Wang, X.; Zhang, X. DEGseq: An R package for identifying differentially expressed genes from RNA-seq data. Bioinformatics 2011, 26, 136-138. [CrossRef] [PubMed]

46. Liu, S.; Chen, J.; Li, S.; Zeng, X.; Meng, Z.; Guo, S. Comparative transcriptome analysis of genes involved in GA-GID1-DELLA regulatory module in symbiotic and asymbiotic seed germination of Anoectochilus roxburghii (Orchidaceae). Int. J. Mol. Sci. 2015, 16, 30190-30203. [CrossRef] [PubMed]

47. Mao, X.; Cai, T.; Olyarchuk, J.G.; Wei, L. Automated genome annotation and pathway identification using the KEGG Orthology (KO) as a controlled vocabulary. Bioinformatics 2005, 21, 3787-3793. [CrossRef] [PubMed]

48. Qian, X.; Sun, L.; Xu, X.; Zhu, B.; Xu, H. Differential expression of VvLOXA diversifies C6 volatile profiles in some Vitis vinifera table grape cultivars. Int. J. Mol. Sci. 2017, 18, 2705-2719. [CrossRef] [PubMed]

49. An, H.; Zhu, Q.; Pei, W.; Fan, J.; Liang, Y.; Cui, Y.; Lv, N.; Wang, W. Whole-transcriptome selection and evaluation of internal reference genes for expression analysis in protocorm development of Dendrobium officinale Kimura et Migo. PLoS ONE 2016, 11, e0163478. [CrossRef] [PubMed]

50. Fochi, V.; Chitarra, W.; Kohler, A.; Voyron, S.; Singan, V.R.; Lindquist, E.A.; Barry, K.W.; Girlanda, M.; Grigoriev, I.V.; Martin, F.; et al. Fungal and plant gene expression in the Tulasnella calospora-Serapias vomeracea symbiosis provides clues about nitrogen pathways in orchid mycorrhizas. New Phytol. 2017, 213, 365-379. [CrossRef] [PubMed]

(c) 2018 by the authors. Licensee MDPI, Basel, Switzerland. This article is an open access article distributed under the terms and conditions of the Creative Commons Attribution (CC BY) license (http:/ / creativecommons.org/licenses/by/4.0/). 